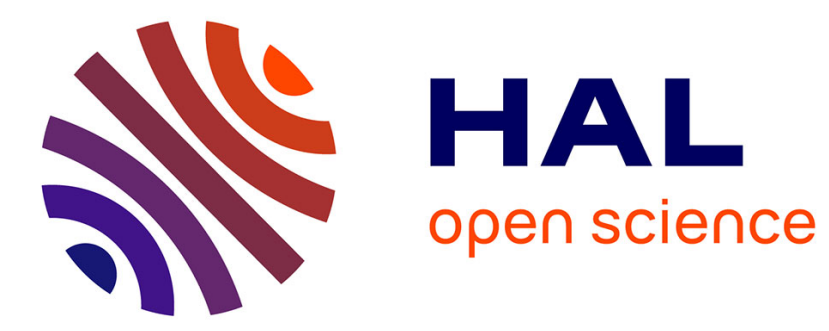

\title{
Smoothing algorithms for mean-flow extraction in large-eddy simulation of complex turbulent flows
}

Adrien Cahuzac, Jérôme Boudet, Pierre Borgnat, Emmanuel Lévêque

\section{To cite this version:}

Adrien Cahuzac, Jérôme Boudet, Pierre Borgnat, Emmanuel Lévêque. Smoothing algorithms for mean-flow extraction in large-eddy simulation of complex turbulent flows. Physics of Fluids, 2010, 22, pp.125104. 10.1063/1.3490063 . hal-00566009

\section{HAL Id: hal-00566009 \\ https://hal.science/hal-00566009}

Submitted on 11 Jun 2012

HAL is a multi-disciplinary open access archive for the deposit and dissemination of scientific research documents, whether they are published or not. The documents may come from teaching and research institutions in France or abroad, or from public or private research centers.
L'archive ouverte pluridisciplinaire HAL, est destinée au dépôt et à la diffusion de documents scientifiques de niveau recherche, publiés ou non, émanant des établissements d'enseignement et de recherche français ou étrangers, des laboratoires publics ou privés. 


\title{
Smoothing algorithms for mean-flow extraction in large-eddy simulation of complex turbulent flows
}

\author{
A. Cahuzac, ${ }^{1, a)}$ J. Boudet, ${ }^{1, b)}$ P. Borgnat, ${ }^{2, c)}$ and E. Lévêque ${ }^{2, d)}$ \\ ${ }^{1}$ LMFA, École Centrale de Lyon, Université de Lyon, CNRS, 36 Avenue Guy de Collongue, \\ 69134 Ecully Cedex, France \\ ${ }^{2}$ Laboratoire de Physique, ENS de Lyon, Université de Lyon, CNRS, 46 allée d'Italie, \\ 69364 Lyon Cedex 07, France
}

(Received 18 February 2010; accepted 20 August 2010; published online 14 December 2010)

\begin{abstract}
Based on physical arguments, the importance of separating the mean-flow from turbulence in the modeling of the subgrid-scale eddy-viscosity is emphasized. Therefore, two distinct time-domain smoothing algorithms are proposed to estimate the mean-flow as the simulation progresses, namely, an exponentially weighted moving average (or exponential smoothing) and an adaptive low-pass Kalman filter. These algorithms highlight the longer-term evolution or cycles of the flow but erase short-term fluctuations. Indeed, it is our assumption that the mean-flow (in the statistical sense) may be approximated as the low-frequency component of the velocity field and that the turbulent part of the flow adds itself to this "unsteady mean." The cutoff frequency separating these two components is fixed according to some characteristic time-scale of the flow in the exponential smoothing, but inferred dynamically from the recent history of the flow in the Kalman filter. In practice, these two algorithms are implemented in large-eddy simulations that rely on a shear-improved Smagorinsky's model. In this model, the magnitude of mean-flow rate of strain is subtracted from the magnitude of the instantaneous rate of strain in the subgrid-scale eddy-viscosity. Two test-cases have been investigated: a turbulent plane-channel flow $\left(\mathrm{Re}_{w}=395\right)$ and the flow past a circular cylinder in the subcritical turbulent regime $\left(\operatorname{Re}_{D}=4.7 \times 10^{4}\right)$. Comparisons with direct numerical simulation and experimental data demonstrate the good efficiency of the whole modeling. This allows us to address nonhomogeneous unsteady configurations without adding significant complication and computational cost to the standard Smagorinsky's model. From a computational viewpoint, this modeling deserves interest since it is entirely local in space. It is therefore adapted for parallelization and convenient for boundary conditions. (C) 2010 American Institute of Physics.
\end{abstract}

[doi:10.1063/1.3490063]

\section{CONTEXT AND MOTIVATIONS}

Large-eddy simulation (LES) is a promising technique that offers an affordable means for the numerical simulation of turbulent flows. ${ }^{1,2}$ Unlike the Reynolds-averaged NavierStokes methods commonly used in the industry, LES gives a direct representation of the large-sized turbulent eddies and their dynamics. It is therefore expected to provide a better description of turbulence impacts. LES has already demonstrated its capabilities in computations of academic buildingblock flows; however, further progress is still required to address realistic complex configurations. ${ }^{3}$ The present work aims at improving this situation. Our guideline is to develop numerical modeling that remains as simple as possible in its formulation but captures the "basic physics," therefore offering an interesting compromise between accuracy and computational cost.

Turbulence that occurs in nature, or in engineering flows, is usually not, even approximately, homogeneous. There are frequent variations of the mean velocity with po-

\footnotetext{
${ }^{a)}$ Electronic mail: adrien.cahuzac@ec-lyon.fr.

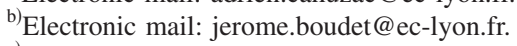

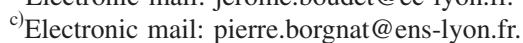

${ }^{\mathrm{d})}$ Electronic mail: emmanuel.leveque@ens-lyon.fr.
}

sition (and time in unsteady configurations). In the following, the general framework of LES is briefly recalled and physical arguments are brought forward to justify the importance of the mean velocity gradients in the modeling of the subgrid-scale (SGS) eddy-viscosity.

LES is rooted in the idea to discretize the flow on a grid whose resolution is coarse compared to the size of the smallest turbulent eddies. Therefore, only the large-sized eddies are represented numerically. This is justifiable since the large-sized eddies contain most of the kinetic energy and their strengths make them the efficient carriers of momentum, heat, mass, etc. On the contrary, the small-sized eddies are mainly responsible for dissipation and contribute little to transport. Conceptually, the solution of a LES is expected to represent the flow variables filtered over a "filter window" whose characteristic width corresponds to the grid resolution. These filtered variables are solutions of the flow equations supplemented by terms accounting for the action of the (unresolved) SGS fluctuations on the grid-scale dynamics. ${ }^{4}$ These additional terms need to be modeled in order to close the governing equations, which constitutes a major difficulty in the case of complex turbulent flows.

In the present study, primarily devoted to weakly compressible aerodynamics, the relevant flow variables are $\rho, \rho \mathbf{u}$, 
and $\rho e_{t}$ referring to the mass, momentum, and total energy of the fluid per unit volume, respectively. The related filtered variables are $\bar{\rho}, \overline{\rho \mathbf{u}} \equiv \bar{\rho} \widetilde{\mathbf{u}}$, and $\overline{\rho e_{t}} \equiv \bar{\rho} \widetilde{e_{t}}$, where the overbar refers to the grid filter operator and the tilde is the Favre operator $(\widetilde{q}=\overline{\rho q} / \bar{\rho})$. The filtered flow equations are expressed as

$$
\begin{aligned}
& \frac{\partial \bar{\rho}}{\partial t}+\frac{\partial\left(\bar{\rho} \tilde{u_{j}}\right)}{\partial x_{j}}=0, \\
& \frac{\partial\left(\bar{\rho} \tilde{u_{i}}\right)}{\partial t}+\frac{\partial\left(\bar{\rho} \tilde{u_{i}} \widetilde{u_{j}}\right)}{\partial x_{j}}=-\frac{\partial \bar{P}}{\partial x_{i}}+\frac{\partial \bar{\tau}_{i j}}{\partial x_{j}}+\frac{\partial \Pi_{i j}^{\mathrm{SGS}}}{\partial x_{j}}, \\
& \frac{\partial\left(\bar{\rho} \tilde{e_{t}}\right)}{\partial t}+\frac{\partial\left[\left(\bar{\rho} \tilde{e_{t}}+\bar{P}\right) \tilde{u_{j}}\right]}{\partial x_{j}} \\
& =\frac{\partial\left[\tilde{u_{i}}\left(\bar{\tau}_{i j}+\Pi_{i j}^{\mathrm{SGS}}\right)\right]}{\partial x_{j}}+\frac{\partial}{\partial x_{j}}\left(\bar{\lambda} \frac{\partial \tilde{T}}{\partial x_{j}}\right)-\frac{\partial \Theta_{j}^{\mathrm{SGS}}}{\partial x_{j}} .
\end{aligned}
$$

Implicit summation on repeated indices is used. The fluid is air, considered as Newtonian with constant dynamic viscosity $\bar{\mu}=1.8 \times 10^{-5} \mathrm{~kg} \mathrm{~m}^{-1} \mathrm{~s}^{-1}$,

$$
\bar{\tau}_{i j}=\bar{\mu}\left(\frac{\partial \tilde{u_{i}}}{\partial x_{j}}+\frac{\partial \tilde{u_{j}}}{\partial x_{i}}-\frac{2}{3} \frac{\partial \tilde{u_{k}}}{\partial x_{k}} \delta_{i j}\right),
$$

and constant thermal conductivity $\bar{\lambda}=2.54$ $\times 10^{-2} \mathrm{~W} \mathrm{~m}^{-1} \mathrm{~K}^{-1}$. Air is assumed to behave as a perfect gas, thus following

$$
\bar{P}=r \bar{\rho} \tilde{T} \quad \text { with } r=287 \mathrm{~J} \mathrm{~kg}^{-1} \mathrm{~K}^{-1} \text {, }
$$

where $P$ is the pressure and $T$ is the temperature. The ratio of specific heats (at constant pressure and volume) is fixed at $\gamma=C_{p} / C_{v}=1.4$. The SGS stress tensor $\left(\Pi_{i j}^{\mathrm{SGS}}\right)$ and the SGS heat current $\left(\Theta_{j}^{S G S}\right)$ encompass the exchanges of momentum and heat with the SGS motions. A common thread is to assume that these terms are essentially responsible for a diffusive transport at grid scale. This assumption yields

$$
\begin{aligned}
& \Pi_{i j}^{\mathrm{SGS}}=\mu_{\mathrm{SGS}}\left(\frac{\partial \tilde{u_{i}}}{\partial x_{j}}+\frac{\partial \tilde{u_{j}}}{\partial x_{i}}-\frac{2}{3} \frac{\partial \tilde{u_{k}}}{\partial x_{k}} \delta_{i j}\right) \text { and } \\
& \Theta_{j}^{\mathrm{SGS}}=\frac{-\mu_{\mathrm{SGS}} C_{p}}{\operatorname{Pr}_{\mathrm{SGS}}} \times \frac{\partial \tilde{T}}{\partial x_{j}},
\end{aligned}
$$

where $\mu_{\text {SGS }}$ represents a SGS eddy-viscosity and $\operatorname{Pr}_{\text {SGS }}=0.9$ may be viewed as a fixed SGS Prandtl number. ${ }^{5}$ Unlike the molecular viscosity, the eddy-viscosity is a property of the flow but not of the fluid. It is therefore expected to depend explicitly on (resolved) flow variables and on the local grid spacing.

In practice, the eddy-viscosity is primarily intended to ensure the correct drain of kinetic energy from the resolved to the unresolved turbulent scales. Tackling this problem from a deterministic viewpoint is mainly out of reach. ${ }^{6} \mathrm{~A}$ statistical approach is rather adopted and an expression of the eddy-viscosity is sought on the basis of statistical hypotheses made on the SGS turbulent scales. ${ }^{7}$ Along this line, physical arguments are now provided to defend the importance of mean-flow gradients in the SGS properties.

Despite the nonhomogeneous nature of most turbulent flows, it is commonly thought that the small-scale properties of turbulence should be considered as locally homogeneous (and isotropic). This hypothesis relies on the idea that small-sized eddies adjust themselves via strong nonlinear interactions, in which all statistical information about the large-scale inhomogeneities is lost. This is the classical phenomenology of Kolmogorov's theory. ${ }^{8}$ Within this picture, it is implicitly assumed (but often forgotten) that the size of the turbulent eddies should be small compared to the lengthscale associated with the mean velocity gradients, which is

$$
L_{\mathrm{s}} \sim \frac{u^{\prime}}{\mathcal{S}}
$$

where $u^{\prime} \equiv \sqrt{\left\langle\left|\mathbf{u}^{\prime}\right|^{2}\right\rangle}$ and $\mathcal{S} \equiv \sqrt{|\nabla\langle\mathbf{u}\rangle|^{2}}$ denote the norms of the fluctuating velocity and of the mean velocity gradient, respectively. Here, angular brackets a priori refer to an ensemble (statistical) average and the fluctuating velocity arises from the Reynolds decomposition: $\mathbf{u}=\langle\mathbf{u}\rangle+\mathbf{u}^{\prime}$. The characteristic scale (7) is obtained by equaling the time-scale associated with the mean velocity gradient (characteristic of the mean-flow distortion) and the turn-over time of turbulent eddies of size $\ell$ (characteristic of turbulent interactions at scale $\ell)$. That is, $1 / \mathcal{S} \sim \varepsilon^{-1 / 3} \ell^{2 / 3}$ for $\ell=L_{\mathrm{s}}$, according to Kolmogorov's theory. ${ }^{8}$ From the averaged Navier-Stokes equations, the mean dissipation rate (per unit mass) $\varepsilon=-\left\langle u_{i}^{\prime} u_{j}^{\prime}\right\rangle$ $\times \partial\left\langle u_{i}\right\rangle / \partial x_{j} \sim u^{\prime 2} \mathcal{S}$, which finally yields $L_{\mathrm{s}} \sim u^{\prime} / \mathcal{S}$ or, equivalently, ${ }^{9} L_{\mathrm{s}} \sim \sqrt{\varepsilon / \mathcal{S}^{3}}$. Note that the previous reasoning relies on dimensional arguments that ignore dimensionless factors. In that sense, the symbol " " should be understood as "is of the order of." In the jargon of fluid mechanics, $\mathcal{S}$ is usually referred to as the shear and $L_{\mathrm{s}}$ is the shear lengthscale. The shear is often expressed as

$$
\mathcal{S}=\sqrt{2\left\langle S_{i j}\right\rangle\left\langle S_{i j}\right\rangle},
$$

where $\left\langle S_{i j}\right\rangle$ is the rate-of-strain tensor of the mean-flow.

Turbulent eddies of size smaller than $L_{\mathrm{s}}$ evolve rapidly enough and are insensitive to the mean-flow distortion: The mean-flow acts principally to convect these eddies without significantly stretching them. On the contrary, turbulent eddies of size larger than $L_{\mathrm{s}}$ have no time to adjust dynamically while they are distorted by the mean-flow gradients. Termed differently, the shear length-scale $L_{\mathrm{s}}$ isolates the problem of the self-interaction of fluctuating velocities, at scales $\ell<L_{\mathrm{s}}$, from the problem of the interaction of the fluctuating velocity with the mean-flow velocity, at scales $\ell>L_{\mathrm{s}}$. This feature is of great importance in the context of LES, as it implies that the statistical properties of the SGS motion (and therefore the nature of the SGS eddy-viscosity) should differ depending on whether the grid resolution $\Delta$ is locally smaller, or larger, than the shear length-scale $L_{\mathrm{s}}$. Therefore, it is desirable to account explicitly for the mean-flow gradients in the modeling of the SGS eddy-viscosity, also the canonical assumption of homogeneous and isotropic turbulence (which ignores the mean-flow inhomogeneities: $\mathcal{S} \simeq 0$ and $L_{\mathrm{s}} \simeq \infty$ ) 
should be abandoned. This is particularly relevant for highReynolds-number flows, when the resolution of the simulation is almost comparable to the large scales of the flow. These features naturally call for a generic procedure to estimate numerically the mean-flow and evaluate the SGS eddyviscosity as the LES progresses. This is the main concern of the present study, which will be developed in Sec. II. Practical implementations in LES relying on a shear-improved Smagorinsky's model will be examined in Sec. III.

\section{SMOOTHING ALGORITHMS FOR MEAN-FLOW EXTRACTION}

Statistical ensemble average may be approximated by space average over directions of homogeneity, whenever it is possible. When it is not, time average may be used instead. Space average is relevant to simple-geometry flows (with directions throughout which fluctuations can be considered as statistically homogeneous), whereas time average is adequate when the flow (its mean and its fluctuations) remains statistically stationary in time. In the presence of deterministic unsteadiness, such as vortex shedding or blade passing in a turbomachine, these two approximations must clearly be abandoned. Therefore, a more general procedure must be designed to extract the mean velocity field and track its possible evolution (if large-scale instabilities develop in the flow).

Our proposal is based on weighted moving average in time, which may be viewed as a generic smoothing operation that highlights longer-term variations or cycles but erase rapid fluctuations. It is here assumed that the mean-flow is given (at each grid point) by the low-frequency component of the velocity field, and that the turbulent component of the flow adds itself to this "unsteady mean." From the viewpoint of signal-processing, extracting a low-frequency trend from a time series is a "dangerous technique" that requires to determine a priori a cutoff frequency above which excitations should be ignored. This cutoff frequency is well defined if there exists a clear-cut separation in the power-spectrum of the signal so that a low-frequency component may be isolated from the high frequencies. Also, it assumes that the separation between the mean-flow and such fluctuations is stationary. In the context of turbulent flows, there is no clear separation in frequency, as fluid dynamics is known to exhibit large-band spectra, 5 and steadiness is not always achieved either. Nevertheless, strategies have been elaborated in order to extract low-frequency trends from the velocity time series. These trends are assumed to be representative of the mean-flow behavior, even if they unavoidably include some turbulent low-frequencies as well.

Two distinct algorithms are considered, based on existing well-known smoothing schemes. They are presented in detail and discussed in relation to their application to meanflow extraction. First, we introduce an exponentially weighted moving average (usually called exponential smoothing) with a fixed smoothing factor, hence a stationary method. This basic method should be considered as a baseline approach, a starting point for comparisons with more elaborated smoothing methods. Then, an adaptive Kalman filter is presented as a refinement of the exponential smoothing. This second approach does not require an a priori stated value for the cutoff frequency but only the prescription of a relevant interval for this frequency; the cutoff frequency adapts itself dynamically within this interval according to the recent history of the signal. These two methods are now presented.

\section{A. Exponentially weighted moving average}

A simple method for extracting the low-frequency component of the velocity field would be a moving (unweighted) arithmetic average of the velocity signal (at each mesh-point) over a time interval of length $\tau_{c}=(N-1) \Delta t$, where $\Delta t$ is the time step of the simulation and $N$ is the number of points retained in the average. This method would correspond to a low-pass filtering with a cutoff frequency $f_{c} \sim 1 / \tau_{c}$. Unfortunately, this simple technique requires the storage of the last $N$ instants in order to maintain a constant $f_{c}$. This appears to be prohibitive in memory when processed over a threedimensional grid with an integration time step typically much smaller than the cutoff period. Another disadvantage is that it cannot be used on the first $N-1$ instants.

These limitations are alleviated by considering an exponentially weighted moving average, also referred to as exponential smoothing in literature and originally introduced in the works of Brown and Holt in the 1950s (see, e.g., the reviews in Refs. 10-12). The main point is to update at each time step the previous estimate of the mean by taking into account the new data point. Let us denote by $[u]^{(n)}$ the estimated mean of one component of the velocity $u$ at time $n$ and at some arbitrary mesh-point. The update of this estimated mean writes in a recurrence form,

$$
[u]^{(n+1)}=\left(1-c_{\exp }\right)[u]^{(n)}+c_{\exp } u^{(n+1)}
$$

with $[u]^{(0)}=u^{(0)}$ at initial time and the smoothing factor $0<c_{\exp }<1$. This algorithm requires only the storage of the mean $[u]$, which is a major improvement over the arithmetic average. The smoothing factor $c_{\exp }$ somehow controls the range of past iterations influencing the estimation of the mean. From Eq. (8), an older data point at instant $m$ contributes to the mean-flow at instant $n+1>m$ with an exponentially decreasing weight $\left(1-c_{\text {exp }}\right)^{n+1-m} \times c_{\text {exp }}$, therefore giving more importance to the recent observations while still not discarding older observations entirely.

To be physically sound, $c_{\exp }$ should be related to the cutoff frequency of this smoothing. In order to exhibit this relation, we shall reformulate Eq. (8) under the form of a digital filter. ${ }^{13}$ First, let us recall the general $z$-transform applied to a discrete-time process $X^{(n)}: X^{(n)} \rightarrow \hat{X}(z)=\Sigma_{n} X^{(n)} z^{-n}$. The $z$-transform applied to Eq. (8) yields

$$
\widehat{[u]}(z)=\frac{c_{\exp }}{1-\left(1-c_{\exp }\right) z^{-1}} \hat{u}(z) .
$$

By considering $z=e^{i \omega \Delta t}$ (the relation between $z$ and the ana$\log$ harmonic frequency $\omega$ when the signal has sampling period $\Delta t$ ), one gets that the power-spectra of $[u]^{(n)}$ and $u^{(n)}$ are linked by the transform 


$$
|\widehat{[u]}(\omega)|^{2}=\frac{c_{\text {exp }}^{2}}{\left|e^{i \omega \Delta t}-\left(1-c_{\exp }\right)\right|^{2}} \cdot|\hat{u}(\omega)|^{2},
$$

which is a discrete-time first-order low-pass filter. The cutoff frequency $f_{c}$, at which the amplitude is reduced by half, ${ }^{14}$ is given by

$$
\frac{c_{\exp }}{\left|e^{i \omega_{c} \Delta t}-\left(1-c_{\exp }\right)\right|}=\frac{1}{2} \quad \text { with } \omega_{c}=2 \pi f_{c} .
$$

Solving directly this equation gives $c_{\exp }=\left(\sqrt{6 \alpha_{c}+\alpha_{c}^{2}}-\alpha_{c}\right) / 3$ with $\alpha_{c}=1-\cos \left(\omega_{c} \Delta t\right)$.

In practical simulations, $\omega_{c} \Delta t \ll 1$ (a necessary condition for time integration) and therefore $c_{\exp } \simeq \omega_{c} \Delta t / \sqrt{3}$, which finally yields

$$
\mathrm{c}_{\exp } \simeq \frac{2 \pi f_{c} \Delta t}{\sqrt{3}} \approx 3.628 f_{c} \Delta t .
$$

This first method acts as a low-pass filtering on the velocity time signal with a fixed cutoff frequency. The main advantage of this method is its simplicity, both conceptually and in its implementation; the computational cost is also very low. However, a known limitation (as already mentioned) is the difficulty to pick an appropriate cutoff frequency for the whole flow. As argued in Ref. 10, this parameter is dangerous to be guessed at. Unavoidably, there is a trade-off between the ability to follow faithfully a quick evolution of the mean, which requires a cutoff frequency (and therefore $c_{\text {exp }}$ ) not too small, and the capacity to smooth out correctly the fluctuations, for which a small cutoff frequency is usually better. Moreover, for a slowly evolving mean-flow, there is an inevitable delay (induced by the exponential smoother) with respect to the proper dynamic. This delay is given by the group delay at null frequency: $:^{13} \Delta t / c_{\exp }$, so the smaller $c_{\text {exp }}$ is, the larger the delay is. These drawbacks restrict the use of the exponential smoothing to "well-characterized" unsteady flows, for which one can single out a characteristic frequency associated with unsteadiness. In order to address arbitrary flows, a more flexible algorithm is required, capable of adapting itself dynamically to the local behavior of the flow. This had led us to design a second algorithm based on the Kalman filtering.

\section{B. Adaptive Kalman filtering}

A more elaborated way to extract the mean velocity field is by use of a Kalman filter. ${ }^{12}$ In brief, a Kalman filter is a recursive filter that estimates the state of a dynamic system, here the mean velocity of the flow, from a series of measurements (or observations). Kalman filtering is a major topic in control theory and control systems in engineering science, and is known to be rather efficient. ${ }^{12,15} \mathrm{An}$ important feature of this filter is its formulation as a recursive estimator, in which the updated state is computed from the previous state and the current measurement only (as for the exponential smoothing). Moreover, being adaptive in nature, the Kalman filter is expected to allow for a better control and adjustment to the low-frequency behavior of the flow, especially in localized regions where instabilities develop (in the vicinity of an obstacle, for instance). From a computational viewpoint, it is therefore an interesting alternative solution to the exponential smoothing.

The problem can be formulated quite simply in a statespace representation, in which the mean velocity (at each mesh-point) represents the state variable of the system and the instantaneous velocity is an observation,

$$
\left\{\begin{array}{c}
{[u]^{(n)}=[u]^{(n-1)}+\delta[u]^{(n)}} \\
u^{(n)}=[u]^{(n)}+\delta u^{(n)} .
\end{array}\right.
$$

This is the state-space representation of a random walk model with noise, ${ }^{12}$ the noise representing here the fluctuations added to the mean-flow. In this form, the mean velocity adjusts itself at each time step with some control increment $\delta[u]^{(n)}=[u]^{(n)}-[u]^{(n-1)}$. The measurement equation expresses the deviation of the instantaneous velocity from the estimated mean: $\delta u^{(n)}=u^{(n)}-[u]^{(n)}$. These two quantities are modeled as random Gaussian processes with zero mean and normal variance. Here, it is assumed that the evolution of the mean velocity is (very) slow compared to the evolution of the instantaneous velocity so that no deterministic evolution is prescribed for $[u]:[u]^{(n)}=[u]^{(n-1)}$ a priori. However, $[u]$ is authorized to evolve (like a sort of biased random walk) if the observation departs significantly from the mean. In our model, the variance of $\delta[u]$ is fixed but the variance of $\delta u$ is updated dynamically as the simulation evolves. This strategy allows the instantaneous velocity to depart significantly from its mean, which is expected to fluctuate in a gentler manner.

The preliminary step is the initialization of the algorithm. Initially, the mean is fixed at $[u]^{(0)}=u^{(0)}$. The root mean square of $\delta[u]$ is kept constant throughout the iterations (as previously mentioned) and fixed at

$$
\sigma_{\delta[u]}=\frac{2 \pi f_{c} \Delta t}{\sqrt{3}} u^{*},
$$

where $u^{*}$ should be interpreted as a "reference velocity" representative of large-scale turbulence (e.g., in a plane-channel flow $u^{*}$ should be identified with the friction velocity at the wall). This essential choice for $\sigma_{\delta[u]}$ stems from the property that in a steady regime for the mean-flow, our Kalman filter should behave as an exponential smoothing with smoothing factor $c_{\exp }=\sigma_{\delta[u]} / \sigma_{\delta u}$ (as briefly demonstrated in Ref. 16). Thus, our expression for $\sigma_{\delta[u]}$ is consistent with the requirement that for stationary periods, $\sigma_{\delta[u]} / \sigma_{\delta u} \simeq 2 \pi f_{c} \Delta t / \sqrt{3}$ [according to Eq. (9)] with $\sigma_{\delta u} \simeq u^{*}$. Finally, the error covariance, which enters in the computation of the optimal Kalman gain (see below), is initialized by $P^{(0)}=\sigma_{\delta u}^{2(0)}$ with $\sigma_{\delta u}{ }^{(0)}=\sigma_{\delta[u]}$.

Standard calculations express a Kalman filter in a recursive form implying two distinct phases: predict and update. The "predict phase" uses the state estimate from the previous timestep to produce a prediction at the current timestep. In the "update phase," measurement information at the current timestep is used to refine this prediction by use of an optimal Kalman gain. Accordingly, the complete algorithm (following the standard theory of the Kalman filtering ${ }^{12,15}$ ) is organized as follows: 
Predict:

- The mean estimate does not a priori change: $[u]^{(n+1)}=[u]^{(n)}$. The error covariance is predicted as $\widetilde{P}^{(n+1)}=P^{(n)}+\sigma_{\delta[u]}^{2}$.

Update:

- The optimal Kalman gain is given by

$$
K^{(n+1)}=\widetilde{P}^{(n+1)} \times \frac{1}{\widetilde{P}^{(n+1)}+\sigma_{\delta u}^{2(n)}}
$$

and the updated mean estimate is obtained by the "correction equation"

$$
[u]^{(n+1)}=\widetilde{[u]^{(n+1)}}+K^{(n+1)} \times\left(u^{(n+1)}-\widetilde{[u]^{(n+1)}}\right) .
$$

This expression is reminiscent of Eq. (8) for the exponential smoothing. Here, the Kalman gain acts as an instantaneous equivalent of the smoothing factor $c_{\text {exp }}$.

- The error covariance is updated (for the next iteration) by

$$
P^{(n+1)}=\widetilde{P}^{(n+1)}-K^{(n+1)} \times \widetilde{P}^{(n+1)} .
$$

Finally, in order to make the Kalman filter adaptive, an estimate of the variance of $\delta u$ is required. For this purpose, we suggest the following formula:

$$
\sigma_{\delta u}^{2(n+1)}=\max \left(\widetilde{\sigma_{\delta u}^{2}}, 0.1 \times u^{* 2}\right),
$$

with

$$
\widetilde{\sigma_{\delta u}^{2}}=u^{*} \times\left|[u]^{(n+1)}-u^{(n+1)}\right| .
$$

The estimator (15) is ad hoc and devised to yield a physically sound estimation of the variance of the fluctuating velocity: $u^{*}$ is fixed while $\left|[u]^{(n+1)}-u^{(n+1)}\right|$ takes into account the observed (instantaneous) fluctuation. The maximum used in Eq. (14) prevents $\sigma_{\delta u}^{2}$ from vanishing because, in that case, the mean estimate would stick to the instantaneous velocity for all subsequent time steps (this is a pitfall of the algorithm). Indeed, if $\sigma_{\delta u}^{2(n)} \simeq 0$ then $K^{(n+1)} \simeq 1$ and $[u]^{(n+1)}$ $\simeq u^{(n+1)}$, consequently $\sigma_{\delta u}^{2(n+1)} \simeq 0$, etc. The value $0.1 \times u^{* 2}$ acts as a (nonzero) lower bound for $\sigma_{\delta u}^{2}$. This bound is reached in regions where the flow is laminar and smooth, and permits to track the onset of turbulence when it occurs: When fluctuations grow, the estimated observation variance $\sigma_{\delta u}^{2(n)}$ increases and $K^{(n+1)}$ decreases. The fluctuating part is therefore well separated from the mean-flow which has lower frequencies. The numerical factor 0.1 is empirical and should not be considered as a proper parameter of the method. Several tests have shown that the efficiency of the whole Kalman filter was not sensitive to the exact value of this factor: 0.1 is just a correct order of magnitude. Generally speaking, one cannot expect to extract a mean component from instantaneous variations without specifying at least a range of time scales potentially relevant for the evolution of the mean component-Eqs. (14) and (15) —and the maximum therein, fulfill this role.

Obvious limitations of our Kalman filter are related to the assumptions made on the process. First, the state-space representation (10) is linear. Second, noises involved in the random walk and in the observation equation are both considered as Gaussian. These assumptions seem unconnected to the physics of turbulent flows, which are known to be strongly nonlinear processes with non-Gaussian statistics. This is undeniable; however, taking into account a potential nonlinear evolution or some non-Gaussian features may neither be a trivial task nor necessarily provide a significant benefit in the present context. From a signal-processing viewpoint, classical developments of the Kalman filter to nonlinear and/or non-Gaussian statistics would be the extended Kalman filter (possibly with unscented transform) ${ }^{17}$ or the particle filter (relying on sequential Monte Carlo estimates ${ }^{18,19}$ ). Particle filters are ruled out because their good performance comes at the expense of an extremely high computational overhead (as compared to the standard Kalman filter). Extended Kalman filters are useful for nonlinear systems and deal with non-Gaussianity (this latter being directly related to the nonlinearity); however, this approach works only if a local linearization of the state-space model is available and if this expansion is relevant. Turbulent flows do not seem to readily correspond to this framework. Our standard Kalman filter may not be the optimal predictor, nevertheless, it should behave relatively well (being the solution of a least-square approach) if the noise distribution is monomodal and is well-characterized by its mean and its variance (first two cumulants). In other terms, the noises involved in Eq. (10) are not required to be exactly Gaussian: It is enough if their distribution is close to a monomodal distribution with fluctuations spread around a given mean. A linear model is also justified by the requirement to separate a mean from fluctuations. Usual approaches, dating back to the Reynolds decomposition, are based on such linear decomposition, even for unsteady flows. Note that the extraction of the mean-flow is done by a linear procedure, however, the mean-flow evolution results from nonlinear dynamics taken into account in the simulation. Another interest in keeping the simple linear formulation is that it readily compares and extends the exponential smoothing in an adaptive way.

We would like now to briefly comment upon applications of temporal filtering in the general context of LES. Time-domain filtering provides an alternative to (conventional) spatial filtering that offers both physically sound and practical advantages. ${ }^{20}$ In practice, temporal filters naturally commute with spatial differential operator; they also remain manageable in case of unstructured or highly stretched grids whereas spatial filters become problematic. Physically, it is thought that filtering the high-frequency content from the frequency spectrum should effectively remove highwavenumber content from the wavenumber spectrum as well. This is the physical idea behind temporally filtered large-eddy simulations. ${ }^{21}$ Recently, Pruett et al. ${ }^{22}$ developed a temporal approximate deconvolution model, which combines time-domain filtering with linear deconvolution in order to estimate the unfiltered velocity components in the SGS stress tensor. The application of temporal smoothing has also been a standard procedure (possibly in addition to spatial averaging) for extracting mean quantities during a simulation, e.g., in Germano et al. ${ }^{23}$ dynamic procedure. In the 
present work, emphasis has been placed on recasting timedomain filtering in a physical form with a particular interest in unsteady turbulent flows. Our main contribution is the introduction of an adaptive Kalman filter, which alleviates the empirical prescription of a fixed cutoff frequency in the filtering procedure.

\section{TEST CASES}

The previous smoothing algorithms have been implemented in practical LES relying on a shear-improved Smagorinsky's model, ${ }^{24}$ in which the mean velocity field is required to evaluate the SGS eddy-viscosity.

\section{A. The shear-improved Smagorinsky's model}

The physical arguments developed in the first section about nonhomogeneous turbulent flows may be formulated analytically by assuming that the shear is uniform (at least locally). By explicating the scale-by-scale energy budget of shear turbulence from the Navier-Stokes equations, Lévêque et $a .^{24}$ showed evidence that the SGS eddy-viscosity should encompass two types of interactions: (i) between the mean velocity gradient and the resolved fluctuating velocities (the rapid part of the SGS fluctuations ${ }^{25}$ ) and (ii) among the resolved fluctuating velocities themselves (the slow part of the SGS fluctuations). The rapid part is related to the large-scale distortion, while the slow part is associated with Kolmogorov's energy cascade. Interestingly, these developments end up with a model for the SGS eddy-viscosity, in which it appears that the shear (the norm of the mean rate-of-strain tensor) should be subtracted from the norm of the instantaneous rate-of-strain tensor,

$$
\mu_{\mathrm{SGS}}=\bar{\rho}\left(C_{s} \Delta\right)^{2}(|\widetilde{S}|-|\langle\widetilde{S}\rangle|),
$$

where $C_{s}=0.18$ is Smagorinsky's constant for homogeneous and isotropic turbulence, ${ }^{2} \Delta$ is the grid spacing (computed as the cubic root of the cell volume), and $|\widetilde{S}| \equiv \sqrt{2 \tilde{S}_{i j}} \tilde{S}_{i j}$. This improvement accounts for the large-scale distortion in regions of strong shear and, at the same time, allows us to recover the standard Smagorinsky's model ${ }^{26}$ in regions of locally homogeneous and isotropic turbulence (at grid scale). Interestingly, the shear-improved Smagorinsky's model (SISM) does not call for any adjustable parameter (besides $C_{s}$ which is fixed at $C_{s}=0.18$ for all turbulent flows) nor $a d$ hoc damping function. It does not use any kind of dynamic adjustment either. First results concerning a plane-channel flow $^{24}$ and a backward-facing step flow ${ }^{27}$ have shown the good predictive capacity of this model, essentially equivalent to the dynamic Smagorinsky's model ${ }^{23}$ but with a computational cost and a manageability comparable to the original Smagorinsky's model.

It is now our motivation to examine how the SISM behaves when implemented in engineering-flow solvers that use coarser grids, rely on lower-order discretization schemes, and are dedicated to complex-geometry unsteady turbulent flows. For this purpose, the smoothing algorithms presented in the previous section are devoted tools to evaluate the mean rate-of-strain tensor in Eq. (16). In practice, computa-

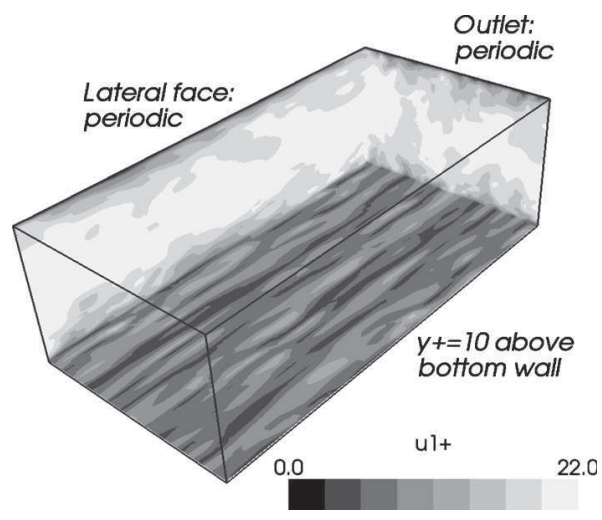

FIG. 1. Instantaneous contours of $u_{1}^{+}$(wall unit streamwise velocity) in the turbulent regime, obtained from the computation using the SISM-ES. As expected, the turbulent flow develops streaky structures in the near-wall region.

tions have been carried out with the solver TURB'FLOW. The dynamical equations have been presented in Sec. I. Spatial discretization is based on finite volumes for multiblock structured grids. Convective fluxes are interpolated with a fourpoint centered scheme (fourth-order on regular grid) and diffusive fluxes with a two-point centered scheme (secondorder). Time stepping relies on a five-step Runge-Kutta scheme. More details about the TURB'FLOW solver may be found in Ref. 28. Two test flows are considered. First is a plane-channel flow configuration ${ }^{2}$ that allows us to isolate the basic features of wall-bounded flows. Second is the flow past a cylinder in subcritical turbulent regime that exhibits phenomena, such as boundary layer separation, transition, vortex shedding, and its interaction with turbulence.

\section{B. Plane-channel flow}

\section{Numerical simulation settings}

The temporal development of a biperiodic plane-channel flow is considered from an initially perturbed laminar flow (Poiseuille's parabolic velocity profile plus a $2 \%$ random relative perturbation). In the fully developed turbulent regime, the objective Reynolds number is $\operatorname{Re}_{w}=\rho u_{w} \delta / \mu=395$ ( $\rho$ is the density, $\delta$ is the channel half width, $u_{w}$ is the mean wall friction velocity, and $\mu$ is the dynamic molecular viscosity). Present LES flow conditions are $\rho=1.214 \mathrm{~kg} \mathrm{~m}^{-3}$ (initial value), $\delta=0.01 \mathrm{~m}$, and $\mu=1.81 \times 10^{-5} \mathrm{~kg} \mathrm{~m}^{-1} \mathrm{~s}^{-1}$ (constant), yielding the objective friction velocity $u_{w}$ $=0.59 \mathrm{~m} \mathrm{~s}^{-1}$. The comprehensive direct numerical simulation (DNS) database obtained by Moser et al. ${ }^{29}$ is used as a reference. An illustration of this test-case is given in Fig. 1.

The mean-flow is oriented along the $x$-direction and plane walls bound the flow at $y= \pm \delta$. Periodicity is imposed in the streamwise and spanwise directions. The computational domain extends over $L_{x} \times L_{y} \times L_{z}=2 \pi \delta \times 2 \delta \times \pi \delta$ (same as the reference DNS). The grid is Cartesian with $49 \times 89 \times 41$ points; a tanh distribution is used in the $y$-direction. In the turbulent regime, the grid spacing is $\Delta x^{+}=52$ (in wall units), $\Delta y^{+}=0.5$ at the wall (11 points below $\left.y^{+}=10\right)$, and $\Delta y^{+}=24$ at centerline, $\Delta z^{+}=31$. This mesh density respects standard recommendations for LES, as re- 
viewed, for example, in Ref. 2. Besides, a very small nondimensional time step, $\Delta t^{+}=\Delta t u_{w} / \delta=3 \times 10^{-5}$, is imposed by the explicit discretization. The pressure is reduced to obtain a Mach number $\mathrm{Ma} \simeq 0.2$ at the centerline, in order to optimize the convergence of the compressible solver, while keeping incompressible flow conditions (typically, throughout the domain at the last instant, density lies within $1 \%$ of the initial density $\rho=1.214 \mathrm{~kg} \mathrm{~m}^{-3}$ ). The momentum in the $x$-direction is maintained by a uniform (over the whole flow) source term that is adjusted dynamically to match the objective mean centerline velocity (calculated as the product of the DNS nondimensional mean centerline velocity and $u_{w}$ $=0.59 \mathrm{~m} \mathrm{~s}^{-1}$ ) on the one hand. The friction velocity is a result of the simulation on the other hand. Actually, for a turbulent velocity profile, the calibration of the mean centerline velocity allows a rather effective calibration of the volume flow rate (or the mass flow rate, given the incompressibility of the flow). This is checked numerically by integrating the mean axial velocity profile with the Simpson rule for nonregular grids. The volume flow rate difference between the different LES simulations and the reference DNS (dimensionalized at the present flow conditions) is lower than $1 \%$. This calibration is consistent with common practice in real flows, where the incident velocity or the volume/mass flow rate is fixed. Moreover, the fixed centerline velocity is a stringent condition when evaluating the description of the wall region.

The numerical results are nondimensionalized with the channel half width, $\delta$, and the objective friction velocity, $u_{w}$. The use of the objective friction velocity (instead of the computed friction velocity) as reference velocity is rather unusual, and will be discussed when addressing the mean velocity profile.

Four LESs have been carried out with different subgridscale models:

- The shear-improved Smagorinsky's model with a spatial average over the $x$ and $z$ directions (SISM-SA).

- The shear-improved Smagorinsky's model with an exponential smoothing (SISM-ES). The smoothing factor $c_{\text {exp }}$ is constant over the whole domain. The cutoff frequency is fixed at $f_{c}=u_{w} / \delta$, which yields $c_{\text {exp }}=1.07 \times 10^{-4}$ from Eq. (9). This cutoff frequency is expected to be representative of the largest eddies and provide a reasonable estimate of the lower bound of the turbulent spectrum.

- The shear-improved Smagorinsky's model with an adaptive Kalman filtering (SISM-AKF). In a consistent way with the exponential smoothing,

$\sigma_{\delta[u]}=\frac{2 \pi f_{c} \Delta t}{\sqrt{3}} u^{*}$

with the characteristic velocity $u^{*}=u_{w}$ and the cutoff frequency $f_{c}=u_{w} / \delta ; \quad u_{w}$ is the objective friction velocity.

- The filtered-structure-function model (FSF). ${ }^{30}$ The most general FSF formulation (known as the sixneighbor formulation) is used. There is no preferred direction in the discretization stencil.
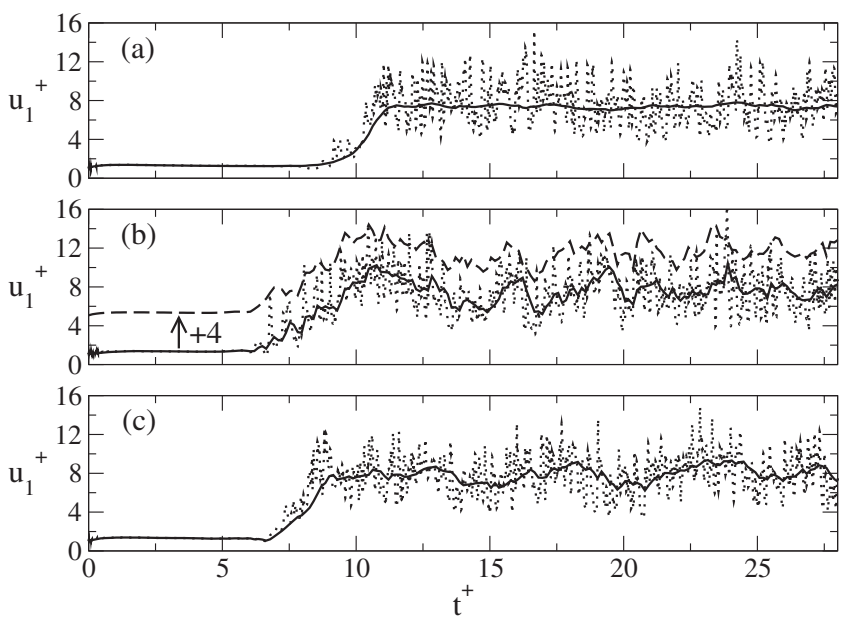

FIG. 2. Numerical velocity probes at $y^{+}=10\left(x=0.19 \times L_{x}\right.$ and $\left.z=0.23 \times L_{z}\right)$ for the simulations with spatial average: SISM-SA (a), exponential smoothing: SISM-ES (b), and adaptive Kalman filtering: SISM-AKF (c). Dots: instantaneous velocity $u_{1}^{+}$; continuous lines: estimated mean velocity $\left[u_{1}^{+}\right]$. The dashed-line in (b) is the postprocessed spatial average along the $x$-direction; this curve has been shifted vertically $(+4)$ for clarity.

\section{Instantaneous velocity probes and smoothing methods}

The different smoothing strategies used for the SISM are illustrated on the velocity signal recorded by a probe at $y^{+}=10\left(x=0.19 L_{x}\right.$ and $\left.z=0.23 L_{z}\right)$. The instantaneous velocity component, $u_{1}^{+}$, is displayed in Fig. 2, together with the mean estimate, $\left[u_{1}^{+}\right]$, resulting from the three different smoothings. The transition to turbulence, characterized by the development of turbulent fluctuations and the increase of the mean velocity, is observed for $6 \leq t^{+} \leq 12$. The high frequencies are obviously suppressed from the smoothed signals. Nevertheless, some low-frequencies persist for the exponential smoothing and the adaptive Kalman filtering as expected from their low-pass behavior. The spatial average [Fig. 2(a)] removes nearly all the fluctuations in the turbulent regime and captures the evolution of the mean velocity from the laminar to the turbulent regime. This average is very efficient indeed, however, it requires spatial homogeneity; a condition which is hardly met in complex flow configurations.

Concerning the exponential smoothing [Fig. 2(b)], the cutoff period was fixed at $T_{c}^{+}=1$. Velocity fluctuations with a period exceeding $T_{c}^{+}$are maintained. Besides, the characteristic time related to the streamwise advection (through the whole channel) may be estimated as $\Delta t_{\text {conv }}^{+}=L_{x}^{+} /\left\langle u_{1}^{+}\right\rangle \simeq 0.82$ at $y^{+}=10$. The exponential smoothing therefore retains dynamical fluid structures whose period is larger than $\Delta t_{\text {conv }}^{+}$. Such events are actually trapped between the periodic streamwise boundary conditions, passing several times in circles through the channel. This feature enforces artificial temporal correlation and weakens the smoothing. This streamwise periodicity is an artifact of the channel-flow configuration that should not occur in practical configurations; the exponential smoothing should then be more regular. For comparison, the spatial average in the $x$-direction is calculated a posteriori. Interestingly, the $x$-average follows quite accurately (with a short time lead) the variations of the 

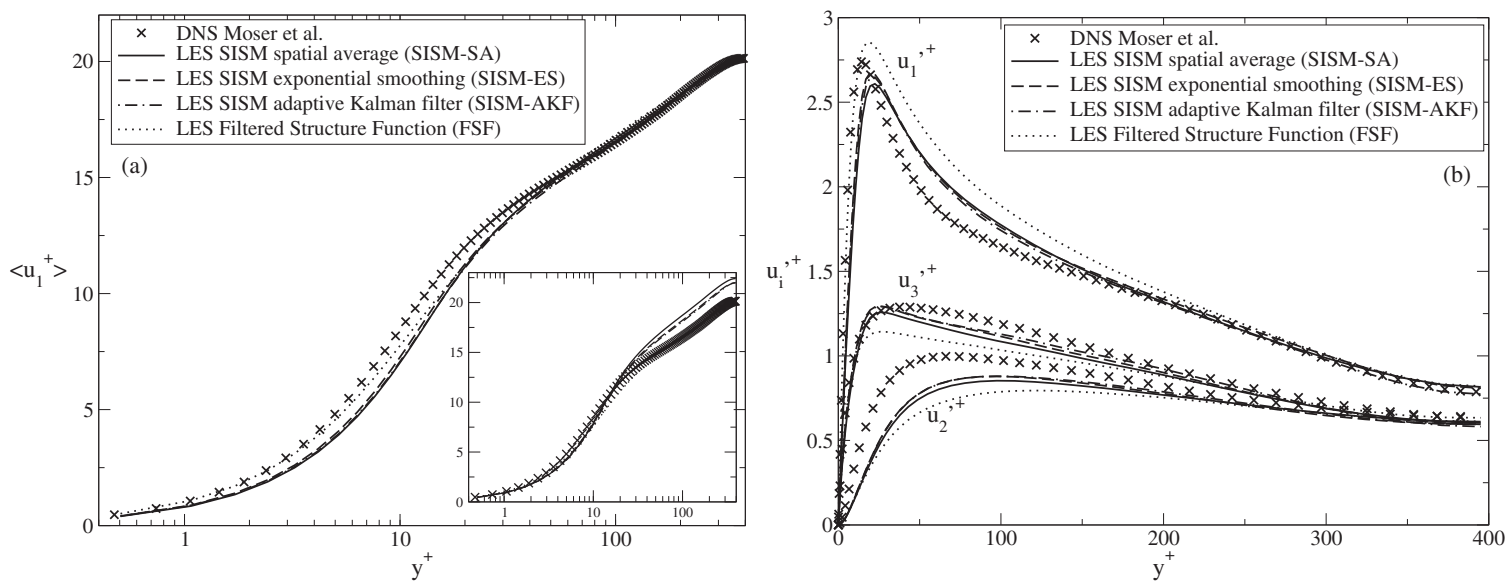

FIG. 3. Mean streamwise velocity profile (a) and turbulent intensity profiles (b). The inlaid graph in (a) presents the mean velocity results scaled by the computed friction velocity (instead of the objective value used for the other plots).

exponential smoothing. This may be explained by the fact that roughly speaking, the whole velocity profile (along the $x$-direction) passes through the probe during a time interval $\Delta t_{\text {conv }}^{+}$of the order of the "memory time," $T_{c}^{+}$. Therefore, the exponential smoothing (in time) is mainly equivalent to the spatial average in the streamwise direction. However, this former does not require any homogeneity conditions. It is local in space and time while the $x$-average relies on a 48 point stencil. Finally, the adaptive Kalman filtering [Fig. 2(c)] behaves qualitatively in a similar way as the exponential smoothing but with a cutoff frequency that adapts itself locally. Here, at $y^{+}=10$, this appears to give smoother results. This feature will be investigated next.

\section{Velocity profiles and energy spectra}

The mean velocity profile and the turbulent intensity profiles (in the stabilized turbulent regime) are displayed in Fig. 3 and compared with DNS data. In the postprocessing, the average (denoted by \langle\rangle ) is meant over space ( $x$ and $z$ directions, $y$-mirror) and time (111 samples for $\left.11.8 \leq t^{+} \leq 28.0\right)$.

In the present paper, the reference velocity for scaling is the objective friction velocity $\left(u_{w}=0.59 \mathrm{~m} \mathrm{~s}^{-1}\right)$. The main graph of Fig. 3(a) is plotted with this convention. However, a graph of the results plotted with a more conventional scaling (based on the computed friction velocity) is inlaid in the major graph, for comparison with literature. The convention of the present paper is preferred because the unified scaling velocity allows a direct comparison between the different versions of LES. More importantly, it makes the nondimensional mean centerline velocity match the reference DNS, which is consistent with the strategy of calibration. In comparison, the centerline error observed in the inlaid graph, using the more conventional scaling, actually comes from the friction velocity error (the mean centerline velocity being imposed). Finally, the present convention allows quantifying the velocity profile quality by considering the friction velocity value.

Considering the major graph of Fig. 3(a), a satisfactory prediction of the mean velocity profile is achieved by the different models. The FSF model yields the best prediction but the different SISM implementations are close, with little influence of the smoothing method. The objective friction velocity $u_{w}$ is $0.59 \mathrm{~m} \mathrm{~s}^{-1}$ (from the reference DNS) compared to $0.60 \mathrm{~m} \mathrm{~s}^{-1}$ for FSF, $0.53 \mathrm{~m} \mathrm{~s}^{-1}$ for SISM-SA, and $0.54 \mathrm{~m} \mathrm{~s}^{-1}$ for SISM-ES and SISM-AKF. In terms of the Reynolds number, these values for the friction velocity yield $\mathrm{Re}_{w}=402$ for FSF, $\mathrm{Re}_{w}=355$ for SISM-SA, and $\mathrm{Re}_{w}=362$ for SISM-ES and SISM-KF, as opposed to $\mathrm{Re}_{w}=395$ for the DNS. Even though the FSF model provides the best estimate, the different SISM implementations lie within $10 \%$ of the objective value, respecting the level of accuracy reviewed in Ref. 31 for standard SGS models. The turbulent intensity profiles are also well-captured for the three velocity components. Here, the SISM provides results closer to the DNS data than the FSF model. Little influence of the smoothing method is again observed.

The spanwise spectra of the streamwise velocity are shown in Fig. 4 for wall-normal distances $y^{+}=10$ and $y^{+}=100$. The LES spectra are in close agreement with the DNS in the range of energy-carrying wavenumbers and the dependence on $y^{+}$is suitably captured. This ability to capture only the large-scale phenomena (with a moderate grid den-

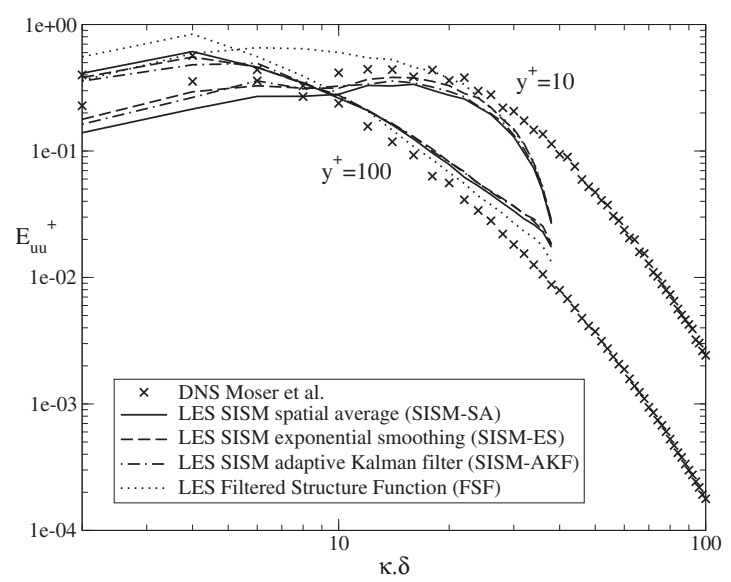

FIG. 4. Spanwise spectra of the streamwise velocity. 

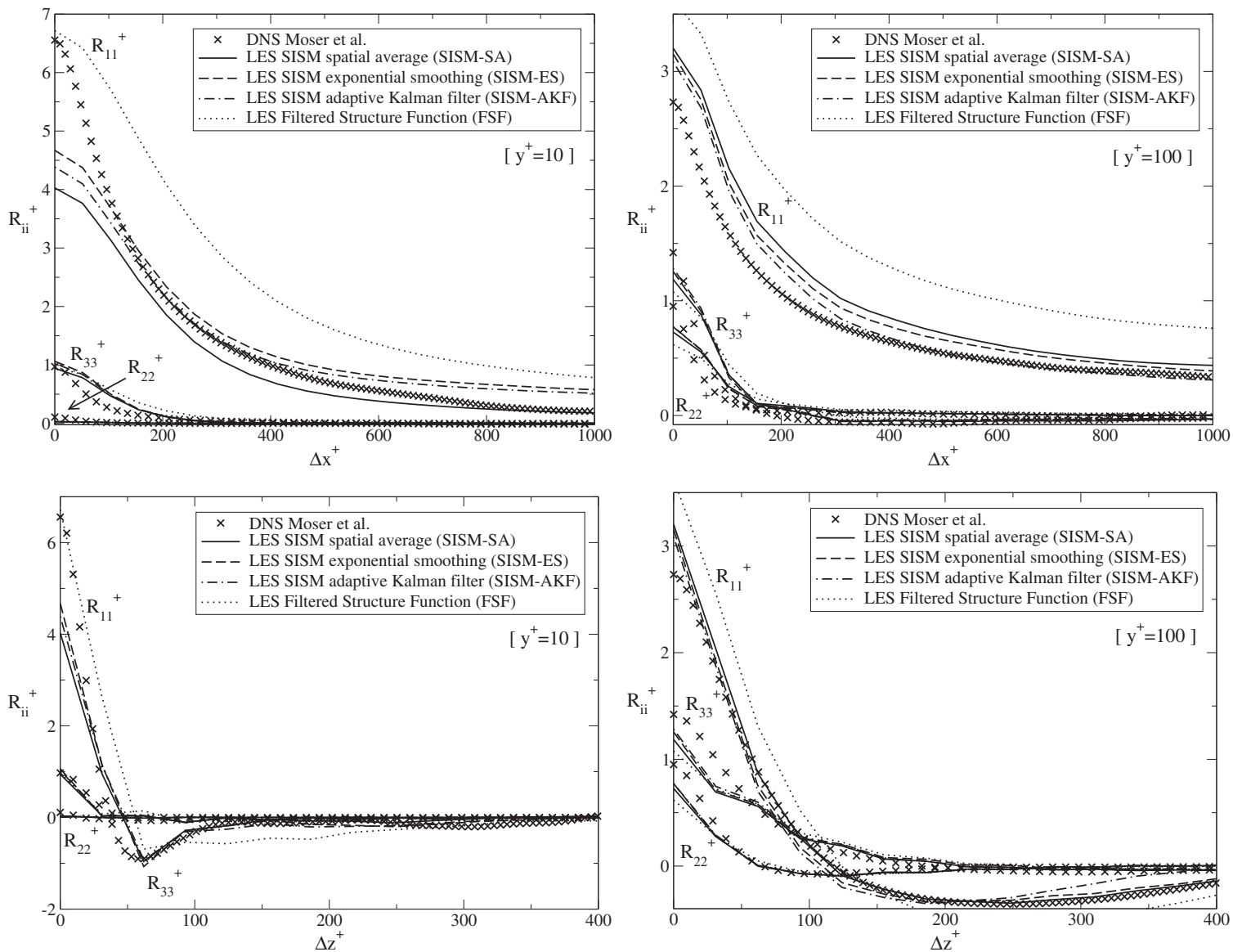

FIG. 5. Two-point velocity autocorrelations for streamwise separations (up) and spanwise separations (down) at $y^{+}=10$ (left) and $y^{+}=100$ (right).

sity) makes the genuine interest, and difficulty as well, of large-eddy simulations. The SISM gives slightly better results than the FSF model (with little influence of the smoothing method).

\section{Two-point velocity autocorrelations}

The two-point velocity autocorrelations in the streamwise and spanwise directions are displayed in Fig. 5 for $y^{+}=10$ and $y^{+}=100$. Interestingly, our SISM implementations appear particularly effective in capturing the correct dependence on the separation distance. Again, the choice of the smoothing method has no noticeable effect. The major trends are fulfilled, that is to say, (i) the correlations spread further in the direction of the flow ( $x$-direction) because of the streaky structures (evidenced in Fig. 1) and (ii) when $y$ increases, correlations spread (in $x$ and $z$ directions) and anisotropy is reduced between the velocity components. In comparison, the FSF model appears slightly less effective in capturing the DNS data. Finally, let us remark that the periodic boundary conditions and the domain extent do not allow for a proper decorrelation in the streamwise direction $\left(L_{x}^{+}=1241\right)$, as evidenced by $R_{11}$ at $y^{+}=100$. This is a known issue of this test flow.

The correlation times $\tau_{i i}^{+}$of the three components of the fluctuating velocity, defined by $\left\langle u_{i}^{\prime}(t) u_{i}^{\prime}\left(t+\tau_{i i}\right)\right\rangle /\left\langle u_{i}^{\prime 2}\right\rangle=1 / 2$ for $i=1,2,3$, are plotted in Fig. 6. Here only, the average $\langle\cdot\rangle$ is meant over space ( $z$ direction and $y$-mirror) and time
(3000 samples for $28.6 \leq t^{+} \leq 29.5$ ). Only the results for the exponential smoothing (SISM-ES) are reported. It is found that the correlation times globally decrease with the distance from the wall and that $\tau_{11}$ (in the streamwise direction) dominates over $\tau_{22}$ and $\tau_{33}$. The cutoff period of the exponential smoothing $\left(T_{c}^{+}=1\right)$ and the mean cutoff period of the Kalman filter (from SISM-AKF) are also displayed in the figure. This latter is obtained by identifying the optimal Kalman gain, $K$, with the smoothing factor of the exponential smoothing and by using Eq. (9) in order to relate this smoothing factor to

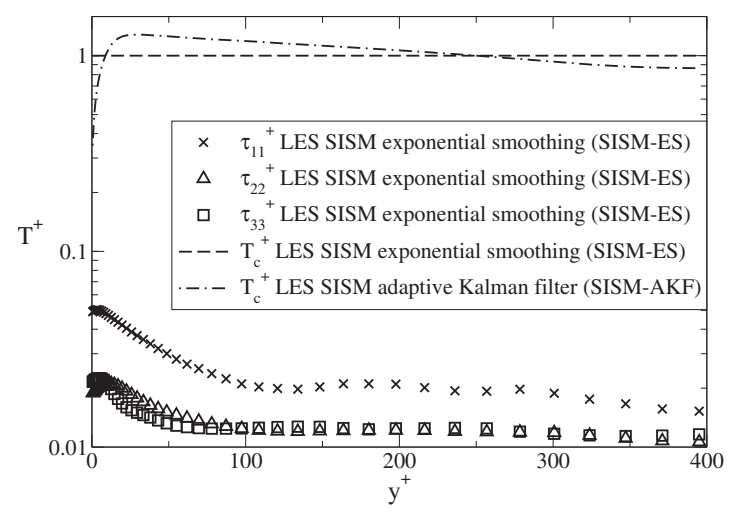

FIG. 6. Correlation times of the three components of the fluctuating velocity and cutoff periods, $T_{c}^{+}$, of the exponential smoothing and the adaptive Kalman filter. 

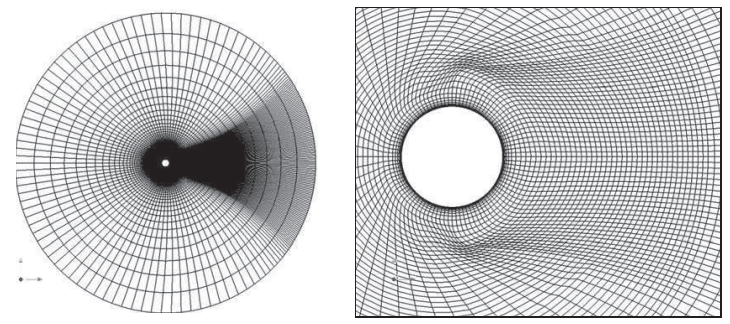

FIG. 7. Cylinder mesh at constant $z$. Left: global view; right: close-up view around the cylinder (every second point in each direction).

$T_{c} \equiv 1 / f_{c}$. This finally yields $T_{c}^{+}=2 \pi \Delta t^{+} / \sqrt{3}\langle K\rangle$, where $\langle\cdot\rangle$ is the usual postprocessing average. The two cutoff periods are found about 20 times larger than the correlation times of the velocity fluctuations, indicating that the two smoothing methods are indeed efficient at filtering out the turbulent fluctuations. The mean cutoff period of the adaptive Kalman filter remains close to 1 but decreases with the wall-normal distance, following the behavior of the velocity correlation times. This is very reasonable since the Kalman filter is expected to adapt its cutoff period according to the observed velocity fluctuations. In this test flow the spatial variation of the correlation times is rather moderate, which explains that the mean cutoff period of the adaptive Kalman filter remains actually very close to the constant cutoff period of the exponential smoothing. This justifies why the two methods mostly behave the same way.

In summary, the results of our SISM-based LES of the channel flow are in good agreement with the reference DNS data for both one- and two-point statistics. The SISM, by explicitly accounting for the mean-flow through a rather simple formulation, reaches predictive qualities similar to the filtered-structure-function model on this test flow. Regarding the extraction of the mean-flow, the exponential smoothing and the adaptive Kalman filter appear to be particularly convenient (computationally sober, local in space) and efficient methods, yielding results essentially similar to the spatial average (in this simple-geometry flow). A more severe test will be considered in the next section.

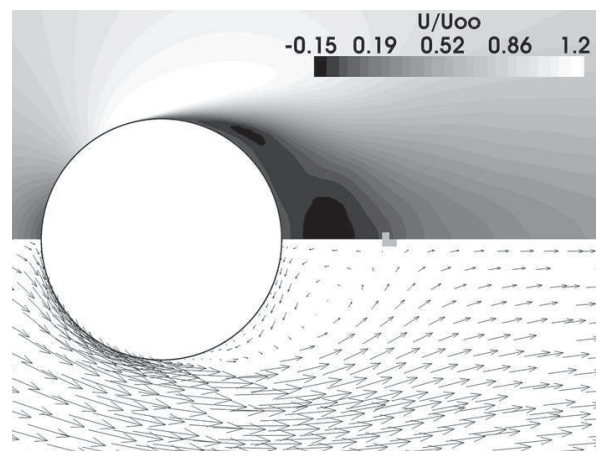

FIG. 8. Mean velocity field obtained from the SISM-ES computation. Up: contours of nondimensional streamwise velocity $\langle U\rangle / U_{\infty}$. Down: velocity vectors $\langle\vec{U}\rangle$. The gray square indicates the end of the recirculation bubble.

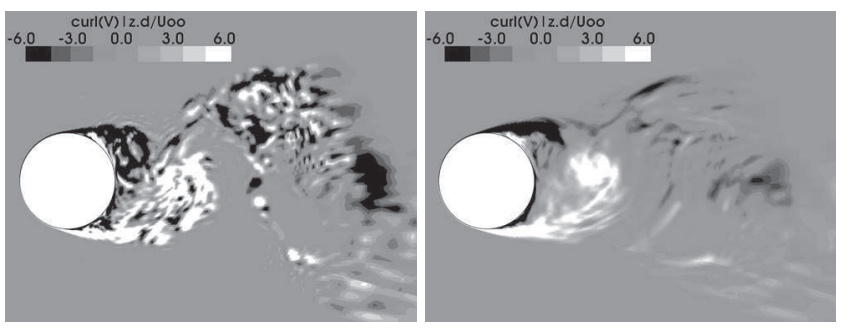

FIG. 9. Nondimensional vorticity field ( $z$ component) from the SISM-ES computation. Left: instantaneous field; right: exponentially smoothed field at the same instant.

\section{Flow past a cylinder in the subcritical turbulent regime}

The numerical simulation of flows around bluff bodies is a great challenge for LES. ${ }^{32}$ In this respect, the flow past a circular cylinder at high Reynolds numbers is of special importance. ${ }^{33}$ The shear-layer transition regime is a welldocumented case that involves complex phenomena and, therefore, deserves interest for our purpose. Here, the definition of the mean-flow requires attention. The boundary layer separation (at the origin of the vortex shedding) occurs upstream the transition, which makes the vortex-shedding part of the (unsteady) mean-flow. Moreover, contrary to the channel flow, there is no homogeneous direction, not even spanwise, because of the three-dimensionality of the vortex shedding. ${ }^{34}$ Therefore, unsteady local smoothing strategies are necessary to extract the mean-flow. Two SISM computations are presented, implementing the exponential smoothing (SISM-ES) and the adaptive Kalman filtering (SISM-AKF), respectively.

\section{Numerical simulation settings}

A circular cylinder of axis $z$ with diameter $D=2 R$ $=0.01 \mathrm{~m}$ is set in an undisturbed airflow with velocity $U_{\infty}$ $=70 \mathrm{~m} \mathrm{~s}^{-1}$ along the $x$-direction (under standard conditions of temperature and pressure). The diameter-based Reynolds number is $\operatorname{Re}_{D}=4.7 \times 10^{4}$, within the subcritical turbulent regime range (more precisely, the shear-layer transition regime). This flow is complex and experiences laminar boundary layer separation, shear-layer transition in the vicinity of the cylinder, turbulent wake, and von Kármán vortex shedding at the Strouhal number $\mathrm{St}=f_{s} D / U_{\infty} \approx 0.2$, yielding $f_{s}$ $\approx 1400 \mathrm{~Hz}$ for the objective vortex-shedding frequency.

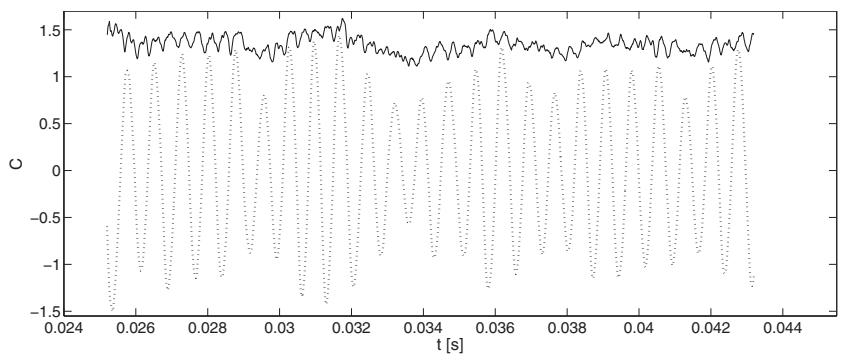

FIG. 10. Temporal evolution of lift and drag coefficients for the SISM-ES computation. (...) $C_{L} ;(-) C_{D}$. 
TABLE I. Force coefficients and Strouhal number for the LES in comparison with experimental data.

\begin{tabular}{lccc}
\hline \hline & SISM-ES & SISM-AKF & Data in literature \\
\hline$\left\langle C_{D}\right\rangle$ : mean drag coefficient & 1.34 & 1.23 & $1.35\left(\operatorname{Re}_{D}=4.3 \times 10^{4}\right)$, Ref. 35 \\
& & {$[1.0,1.35]\left(\operatorname{Re}_{D}=4.8 \times 10^{4}\right)$, Ref. 38} \\
& & {$[1.0,1.3]\left(\operatorname{Re}_{D}=4.8 \times 10^{4}\right)$, Ref. 39} \\
& & {$[1.1,1.3]\left(\operatorname{Re}_{D} \in\left[10^{4}, 10^{5}\right]\right)$, Ref. 37} \\
& & \\
$C_{D}^{\prime}$ : rms drag coefficient & 0.065 & $0.16\left(\operatorname{Re}_{D}=4.3 \times 10^{4}\right)$, Ref. 35 \\
& & {$[0.08,0.1]\left(\operatorname{Re}_{D}=4.8 \times 10^{4}\right)$, Ref. 40} \\
& & {$[0.05,0.1]\left(\operatorname{Re}_{D} \in\left[10^{4}, 10^{5}\right]\right)$, Ref. 37} \\
& & & \\
$C_{L}^{\prime}$ : rms lift coefficient & 0.603 & {$[0.45,0.55]\left(\operatorname{Re}_{D}=4.3 \times 10^{4}\right)$, Ref. 35} \\
& & {$[0.4,0.8]\left(\operatorname{Re}_{D}=4.8 \times 10^{4}\right)$, Ref. 40} \\
& & {$[0.6,0.82]\left(\operatorname{Re}_{D} \in\left[10^{4}, 10^{5}\right]\right)$, Ref. 37} \\
St: Strouhal number & & {$[0.18,0.2]\left(\operatorname{Re}_{D}=4.8 \times 10^{4}\right)$, Ref. 38} \\
& & & {$[0.185,0.195]\left(\operatorname{Re}_{D}=6.1 \times 10^{4}\right)$, Ref. 41} \\
\hline \hline
\end{tabular}

The aspect ratio of the cylinder (span length over radius) has some influence on the flow, ${ }^{35}$ which explains partly the dispersion of the experimental results at comparable Reynolds numbers. In our LES, the grid extends over 3D in the spanwise direction ( $z$-direction) but periodicity is imposed on the end planes so that the computations can be representative of higher aspect ratios. Views of the grid are displayed in Fig. 7. The computational domain extends over 10D in the radial direction and the whole grid uses $3 \times 10^{6}$ points. In the turbulent region, behind the separation line, the wall grid density (in wall units) is $\Delta r_{\text {max }}^{+} \simeq 1$ (with a radial growth ratio of 1.2), $R \Delta \theta_{\max }^{+} \simeq 20$, and $\Delta z_{\max }^{+} \simeq 25$. Again, this mesh density respects standard recommendations for LES. ${ }^{2}$ The nondimensional time step is $\Delta t^{+}=\Delta t U_{\infty} / D=4 \times 10^{-4}$, adapted to explicit discretization.

The SISM-ES computation relies on a fixed smoothing factor $c_{\text {exp }}=6.093 \times 10^{-4}$ (throughout the whole domain). This value of $c_{\exp }$ is obtained from Eq. (9) with $f_{c}=2 f_{s}$, where $f_{s} \approx 1400 \mathrm{~Hz}$ is the objective vortex-shedding frequency. The cutoff frequency is fixed at twice the vortexshedding frequency (the factor 2 is arbitrary) in order to ensure that the vortex shedding is suitably captured in the mean-flow reconstruction. The SISM-AKF computation requires for calibration a reference velocity, $u^{*}$, and a reference frequency, $f_{c}$ [cf. Eqs. (11) and (14)]. Our natural choices are $u^{*}=U_{\infty}$ and $f_{c}=f_{s}$. Contrary to the exponential smoothing, in which $f_{c}$ represents the actual cutoff frequency, $f_{c}$ is here a reference value pointing out the order of magnitude of the cutoff frequency. This latter adapts itself to the local behavior of the flow according to the Kalman filtering procedure. Finally, the computations have been recorded over 24 periods, representing nearly 3000 samples in the established turbulent regime. $\langle\cdot\rangle$ denotes the postprocessing time and spanwise average.

\section{Flow field, forces, and Strouhal number}

An overview of the flow resulting from the SISM-ES computation is presented at first. The mean streamwise ve- locity, $\langle U\rangle$, is represented in the upper half of Fig. 8. The gray square dot delimits the recirculation bubble: the "wakeclosure length" $l_{c}$ is given by $\langle U\rangle\left(x=l_{c}, y=0\right)=0$. In the lower half of the figure, the mean velocity vector field $\langle\vec{U}\rangle$ is displayed. The mean recirculation zone can be clearly identified. In Fig. 9, the z-components of vorticity for both the instantaneous flow and the exponentially smoothed flow are compared at the same instant. While the instantaneous vorticity exhibits a wide range of structure sizes, the exponential-smoothed flow mostly captures the vortex shedding (where shear effects are significant), as expected. Figure 10 displays the temporal evolution of the lift coefficient, $C_{L}$, and drag coefficient, $C_{D}$, over 24 periods of vortex shedding. As already mentioned in Refs. 36 and 37, a strong correlation is observed between the variations of $C_{D}$ and the modulation of $C_{L}$; typically, the value of the drag is high when the lift amplitude is maximum.

These first qualitative results are very satisfactory. Here, only the results with exponential smoothing are shown, but very similar features have been observed for the SISM computation with the adaptive Kalman filter.

Quantitative comparisons are now carried out between the SISM-ES, the SISM-AKF, and available experimental data (at comparable Reynolds numbers). The mean drag coefficient, $\left\langle C_{D}\right\rangle$, the root-mean-square drag coefficient, $C_{D}^{\prime}$, and the root-mean-square lift coefficient, $C_{L}^{\prime}$, are gathered in Table I. While the two smoothing methods had yielded very similar results for the channel-flow computations, notable differences are obtained here. However, both computations give forces that lie within the experimental ranges.

The vortex shedding has a strong impact on the pressure distribution around the cylinder and, therefore, on the lift and drag oscillations. Except from the rearmost part of the cylinder, the wall pressure spectrum is peaked around the shedding frequency, $f_{s}$. From the wall pressure spectrum at $\theta=90^{\circ}(\theta$ is the angle taken from the upstream stagnation point), the vortex-shedding frequency has been identified: 


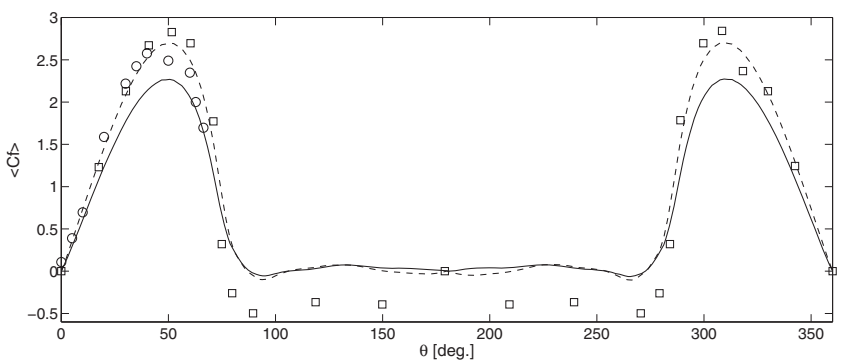

FIG. 11. Circular cylinder skin friction coefficient. (-) SISM-ES at $\mathrm{Re}_{D}=4.7 \times 10^{4} ;(--)$ SISM-AKF at $\operatorname{Re}_{D}=4.7 \times 10^{4} ;(\bigcirc)$ experimental data (Ref. 42) at $\operatorname{Re}_{D}=9.1 \times 10^{4}$; ( $\square$ ) experimental data (Ref. 39) at $\operatorname{Re}_{D}=10^{5}$.

- SISM-ES computation: $f_{s}=1330 \mathrm{~Hz}$ corresponding to a Strouhal number $\mathrm{St}=0.190$;

- SISM-AKF computation: $f_{s}=1422 \mathrm{~Hz}$ corresponding to $\mathrm{St}=0.204$.

In Table I, this key criterion is shown to be in very good agreement with the available experimental data.

\section{Wall friction and pressure distribution}

Figure 11 shows the distribution of the mean friction coefficient, $\left\langle C_{f}\right\rangle \equiv\left[\left\langle\tau_{w}\right\rangle / \frac{1}{2} \rho U_{\infty}^{2}\right] \sqrt{\operatorname{Re}_{D}}$ (where $\tau_{w}$ is the friction), around the cylinder. Both computations are in good agreement with the experimental data, with however a slightly better matching for the SISM-AKF. The mean separation angle, $\theta_{s}$, is the angle for which $\left\langle C_{f}\right\rangle$ vanishes. Here, the measured values are $\theta_{s}=88^{\circ}$ for the SISM-ES computation and $\theta_{s}=86^{\circ}$ for the SISM-AKF computation (with an angular resolution of $\Delta \theta=2^{\circ}$ in this region). According to experimental data ${ }^{37}$ in the range $4.0 \times 10^{4} \leq \operatorname{Re}_{D} \leq 4.5 \times 10^{4}$, the expected separation angle is $\theta_{s} \approx 83^{\circ}$. The LES computations appear to slightly overestimate $\theta_{s}$ but the discrepancy is only two grid points.

In the channel-flow simulation, the cutoff frequency for the exponential smoothing had been evaluated by $f_{c}=u_{w} / \delta$, with $\delta$ being the half width (reference length of the flow) and $u_{w}$ the friction velocity (reference velocity based on the shear at the wall). Along the same line of idea, a cutoff frequency may be designed here from the radius of the cylinder $(R=D / 2)$ and the maximal value of the skin friction velocity on the cylinder, that is,

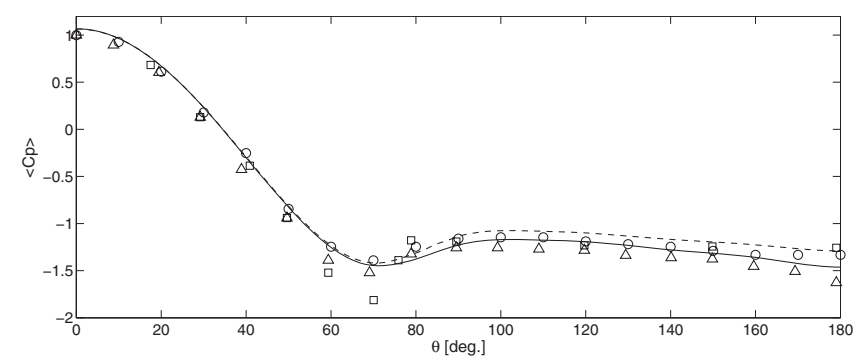

FIG. 12. Mean pressure-coefficient distribution, $\left\langle C_{p}\right\rangle$, around the cylinder. (-) SISM-ES at $\operatorname{Re}_{D}=4.7 \times 10^{4} ;(---)$ SISM-AKF at $\operatorname{Re}_{D}=4.7 \times 10^{4}$; $(\triangle)$ experimental data (Ref. 35) at $\operatorname{Re}_{D}=4.0 \times 10^{4}$; $(\bigcirc)$ experimental data (Ref. 43) at $\operatorname{Re}_{D}=4.6 \times 10^{4} ;(\square)$ experimental data (Ref. 39) at $\operatorname{Re}_{D}=10^{5}$.

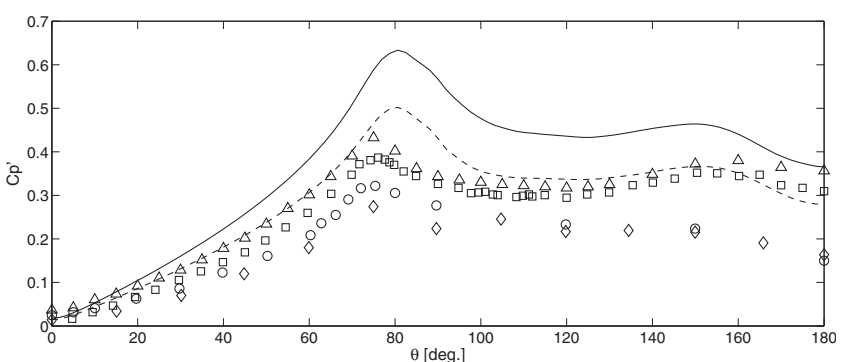

FIG. 13. Root-mean-square pressure-coefficient, $C_{p}^{\prime}$, around the cylinder. (一) SISM-ES at $\mathrm{Re}_{D}=4.7 \times 10^{4} ;(---)$ SISM-AKF at $\operatorname{Re}_{D}=4.7 \times 10^{4}$; $(\square)$ experimental data (Ref. 41) at $\operatorname{Re}_{D}=6.1 \times 10^{4} ;(\triangle)$ experimental data (Ref. 36) at $\operatorname{Re}_{D}=6.1 \times 10^{4} ;(\bigcirc)$ experimental data (Ref. 42) at $\operatorname{Re}_{D}=10^{5}$; $(\diamond)$ experimental data (Ref. 44) at $\operatorname{Re}_{D}=10^{5}$.

$$
f_{\text {shear }}=\frac{\max \left(u_{w}\right)}{R} .
$$

Numerically, this yields $f_{\text {shear }}=1430 \mathrm{~Hz}$ in the SISM-ES computation. This frequency is expected to be representative of the largest turbulent eddies detaching from the cylinder. Interestingly, this is almost equal to the vortex-shedding frequency $\left(f_{s} \approx 1400 \mathrm{~Hz}\right)$ characterizing the mean-flow unsteadiness. The proximity between the frequency associated with the flow unsteadiness $\left(f_{s}\right)$ and the frequency of the largest turbulent eddies $\left(f_{\text {shear }}\right)$ indicates that the mean-flow reconstruction using time-domain filtering necessarily includes (to some extent) the dynamics of large-sized energy-carrying turbulent eddies. One may evaluate that our mean-flow reconstruction encompasses turbulent velocity fluctuations within the frequency band $f_{\text {shear }}<f<f_{c} \approx 2 f_{\text {shear }}$ (since $f_{s} \approx f_{\text {shear }}$ ). This is an unavoidable limitation of our method (already mentioned in Sec. II). However, the present results show that this limitation does not seem to dramatically impact on the quality of the whole modeling. A similar reasoning can be followed for the adaptive Kalman filtering. In that case, the cutoff frequency remains of the order of $f_{\text {shear }}$ and adapts itself dynamically to the fluctuations of the flow. This strategy appears to yield better results.

The distribution of the mean pressure-coefficient, $\left\langle C_{p}\right\rangle$ $\equiv\left(\langle p\rangle-p_{\infty}\right) / \frac{1}{2} \rho U_{\infty}^{2}$, around the cylinder is shown in Fig. 12 and compared with various experimental data. The prediction is very satisfactory for both SISM computations.

Finally, the distribution of the root-mean-square pressure-coefficient, $C_{p}^{\prime}$, is plotted in Fig. 13. The overall behavior is well-captured with a maximum around the point of separation. ${ }^{42}$ The intensity is notably overestimated (about $50 \%$ on the peak level) by the SISM computation with exponential smoothing but the computation with the adaptive Kalman filtering captures much more accurately the experimental levels (particularly those closer to the simulated $\operatorname{Re}_{D}=4.7 \times 10^{4}$ ). This is another illustration of the influence of the smoothing approach on this test-case.

\section{Wake-centerline mean velocity}

Figure 14 displays the mean velocity $\langle U\rangle$ along the wake centerline for both SISM computations, in comparison with the experimental results reported in Ref. 38 at $\mathrm{Re}_{D}=1.4$ 


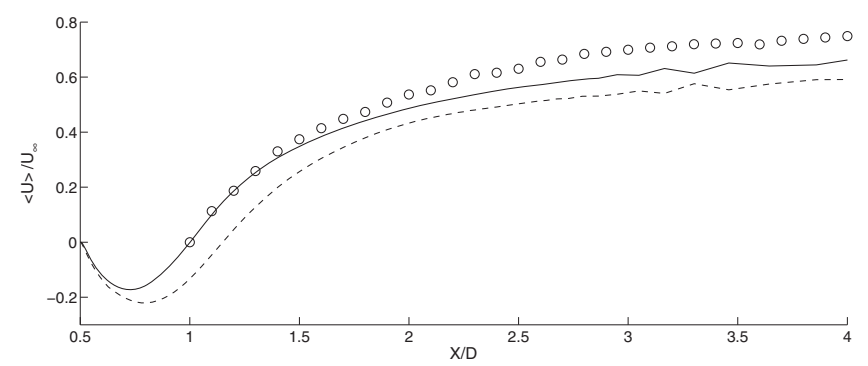

FIG. 14. Mean velocity $\langle U\rangle$ along the wake centerline. (-) SISM-ES at $\operatorname{Re}_{D}=4.7 \times 10^{4} ;(---)$ SISM-AKF at $\operatorname{Re}_{D}=4.7 \times 10^{4} ;(\bigcirc)$ experimental data (Ref. 38) at $\operatorname{Re}=1.4 \times 10^{5}$.

$\times 10^{5}$. The behavior and levels are globally captured. A specific attention can be drawn on the mean separation bubble length (or wake-closure length), $l_{c}$, given by $\langle U\rangle\left(x=l_{c}, y\right.$ $=0)=0$. In this regime, $l_{c}$ is expected to lower with increasing Reynolds number. ${ }^{45}$ For the present LES with $\mathrm{Re}_{D}=4.7$ $\times 10^{4}$, it should to be close to $l_{c} \simeq 1.25 D$. The SISM-ES computation yields $l_{c}=D$, surprisingly close to the value of the experimental profile at $\operatorname{Re}_{D}=1.4 \times 10^{5}$. A much better prediction is again achieved by the SISM-AKF with $l_{c}=1.15 D$.

In summary, LES of the flow past a circular cylinder at $\operatorname{Re}_{D}=4.7 \times 10^{4}$ was carried out using the SISM with two different mean-flow extraction strategies: An exponential smoothing (a baseline method) and an adaptive Kalman filter (an elaborated method). A good overall prediction of this complex flow was achieved for both smoothing methods. However, when using the exponential smoothing some discrepancies have been observed on the mean friction, wall pressure fluctuations, and wake-centerline mean velocity. All these discrepancies are reduced by the use of the Kalman filter. This suggests the necessity of using adaptive (nonhomogeneous) cutoff properties when treating complex (nonhomogeneous) turbulent flows.

\section{SUMMARY AND CONCLUSION}

The physical idea which underlies this study is to take into account the mean-flow inhomogeneities in the subgridscale viscosity. This feature is clearly encompassed in the shear-improved Smagorinsky's model. Numerically, two distinct smoothing algorithms (an exponentially weighted moving average and an adaptive Kalman filter) are proposed to extract the mean-flow as the simulation progresses. Our results indicate that the whole modeling offers an equitable compromise between the accuracy of the numerical solution and the computational cost. Since our method exploits the temporal discretization recurrently, it is entirely local in space and, therefore, suitable for parallelization and convenient for boundary conditions.

In the classical channel-flow test-case, this approach was shown to give satisfactory results regarding both mean and fluctuating velocities, spectra, and two-point correlations. Predictive capabilities appear comparable to the well-known filtered-structure-function model, used as reference. Further improvement of the present approach is still possible, as revealed by the channel configuration through the underpredic- tion of friction velocity. Since the different smoothing strategies, i.e., the reference spatial average and the presently introduced temporal digital filters, appear to achieve very similar results, we believe that major benefit would rather be gained from the improvement of the (numerical) discretization scheme and the subgrid-scale modeling (the shearimproved Smagorinsky's model). The subcritical turbulent flow past a cylinder has provided a more selective case for the smoothing methods, combining both nonhomogeneity and mean-flow unsteadiness. Extensive comparisons have been carried out with experimental data (at comparable Reynolds numbers), concerning the global fluid forces acting on the cylinder, the vortex-shedding frequency, the mean skin friction, the mean and fluctuating pressure distribution (around the cylinder), and the mean wake-centerline velocity. The numerical results using the exponential smoothing exhibit some discrepancies (with experimental data) that are all reduced by using the adaptive Kalman filter. This latter yields a very satisfactory description of the flow characteristics. The adaptivity and efficiency of this modeling (shearimproved Smagorinsky's model in combination with the adaptive Kalman filter) make it very relevant for LES of complex turbulent flows. Especially, applications to proper turbomachine flows are of actual interest.

In the Kalman filtering framework, introducing correlations in time (in order to better smooth the turbulent fluctuations) is readily possible. However, this would significantly increase the computational cost and memory requirements (if several time steps need to be stored). For that reason, this aspect was left out in the present work. Nevertheless, a possible improvement may consist in treating the temporal smoothing from a Lagrangian viewpoint (along fluid trajectories). This is under investigation. Finally, the smoothing approaches investigated in the present work do not a priori require a grid-convergence analysis because they are purely temporal. However, since filtering acts on nonlinear turbulent quantities, which are definitively dependent on the spatial mesh resolution, a parametric study (as a function of the mesh resolution) would certainly deserve interest as well.

\section{ACKNOWLEDGMENTS}

The authors would like to thank J.-P. Bertoglio, P. Flandrin, L. Shao, and F. Toschi for long-term valuable discussions. The numerical simulations rely on the TURB'FLOW solver, originating from the LMFA at the Ecole Centrale de Lyon and codeveloped with the FLUOREM company (France). Simulations have been performed by using the local computing facilities at LMFA and ENS-Lyon (PSMN), and national HPC resources of CINES (Montpellier, France).

\footnotetext{
${ }^{1}$ M. Lesieur, O. Métais, and P. Comte, Large-Eddy Simulations of Turbulence (Cambridge University Press, Cambridge, 2005).

${ }^{2}$ P. Sagaut, Large Eddy Simulation for Incompressible Flows: An Introduction, 3rd ed. (Springer-Verlag, Berlin, 2006).

${ }^{3}$ U. Piomelli, "Large-eddy simulation: Achievements and challenges," Prog. Aerosp. Sci. 35, 335 (1999).

${ }^{4}$ This argument holds if neglecting noncommutation errors between the filter and partial derivatives.

${ }^{5}$ S. B. Pope, Turbulent Flows (Cambridge University Press, New York, 2000).

${ }^{6} \mathrm{C}$. Meneveau and J. Katz, "Scale-invariance and turbulence models for
} 
large-eddy simulation,” Annu. Rev. Fluid Mech. 32, 1 (2000).

${ }^{7}$ J.-P. Chollet and M. Lesieur, "Parametrization of small scales of threedimensional isotropic turbulence utilizing spectral closures," J. Atmos. Sci. 38, 2747 (1981).

${ }^{8}$ U. Frisch, Turbulence: The Legacy of A. N. Kolmogorov (Cambridge University Press, Cambridge, 1995).

${ }^{9}$ H. Tennekes and J. L. Lumley, A First Course in Turbulence (MIT Press, Cambridge, 1972).

${ }^{10}$ E. Gardner, "Exponential smoothing: The state of the art," J. Forecast. 4, 1 (1985).

${ }^{11}$ E. S. Gardner, Jr., "Exponential smoothing: The state of the art-Part II," Int. J. Forecast. 22, 637 (2006).

${ }^{12}$ A. Harvey, Forecasting, Structural Time Series Model and the Kalman Filter (Cambridge University Press, Cambridge, 1989).

${ }^{13}$ A. Papoulis, Signal Analysis (McGraw-Hill, New York, 1984).

${ }^{14}$ Standard usage is to define the cutoff frequency at $-3 \mathrm{~dB}$ related to the attenuation of the power by half, and not of the amplitude. This would result in the removal of $\sqrt{3}$ in Eq. (9), therefore leading to the same order of magnitude for $f_{c}$. Here, we want to follow the convention commonly used in numerical simulations (Ref. 46).

${ }^{15}$ P. J. Brockwell and R. A. Davies, Time Series: Theory and Methods, Springer Series in Statistics, 2nd ed. (Springer, New York, 1991).

${ }^{16}$ In the steady regime, the results in Ref. 12 using the Riccati equations for the Kalman filter show that the Kalman filter used here behaves as $[u]^{(n)}=(1-\lambda)[u]^{(n-1)}+\lambda u^{(n)}$ for large times $n$. The smoothing factor $\lambda$ is given by $\lambda=\bar{P} /(1+\bar{P})$ with $\bar{P}=\lim _{n \rightarrow \infty} P(n \mid n-1)=q+\sqrt{q^{2}+4 q} / 2$ and $q=\sigma_{\delta[u]}^{2} / \sigma_{\delta u}^{2}$. In our physical context, $\lambda \ll 1$ and $\lambda \approx \sqrt{q}=\sigma_{\delta[u]} / \sigma_{\delta u}$. Hence, the rule proposed in the text.

${ }^{17}$ S. J. Julier and J. K. Uhlmann, "Unscented filtering and nonlinear estimation,” Proc. IEEE 92, 401 (2004).

${ }^{18}$ N. J. Gordon, D. J. Salmond, and A. F. M. Smith, "Novel approach to nonlinear/non-Gaussian Bayesian state estimation," IEE Proc. F, Radar Signal Process. 140, 107 (1993).

${ }^{19}$ S. Arulampalam, S. Maskell, N. Gordon, and T. Clapp, "A tutorial on particle filters for on-line nonlinear/non-Gaussian Bayesian tracking," IEEE Trans. Signal Process. 50, 174 (2002).

${ }^{20}$ C. D. Pruett, "Eulerian time-domain filtering for spatial large-eddy simulation," AIAA J. 38, 1634 (2000).

${ }^{21}$ C. D. Pruett, T. B. Gatski, C. E. Grosch, and W. D. Thacker, "The temporally filtered Navier-Stokes equations: Properties of the residual stress," Phys. Fluids 15, 2127 (2003).

${ }^{22}$ C. D. Pruett, B. C. Thomas, C. E. Grosch, and T. B. Gatski, "A temporal approximate deconvolution model for large-eddy simulation," Phys. Fluids 18, 028104 (2006).

${ }^{23}$ M. Germano, U. Piomelli, P. Moin, and W. H. Cabot, “A dynamic subgridscale eddy-viscosity model," Phys. Fluids A 3, 1760 (1991).

${ }^{24}$ E. Lévêque, F. Toschi, L. Shao, and J.-P. Bertoglio, "Shear-improved Smagorinsky model for large-eddy simulation of wall-bounded turbulent flows," J. Fluid Mech. 570, 491 (2007).

${ }^{25}$ L. Shao, S. Sarkar, and C. Pantano, "On the relationship between the mean flow and subgrid stresses in large eddy simulation of turbulent shear flows," Phys. Fluids 11, 1229 (1999).
${ }^{26}$ J. S. Smagorinsky, "General circulation experiments with the primitive equations: I. The basic experiment,” Mon. Weather Rev. 91, 99 (1963).

${ }^{27}$ F. Toschi, H. Kobayashi, U. Piomelli, and G. Iaccarino, "Backward-facing step calculations using the shear improved Smagorinsky model," Proceedings of the Summer Program 2006 (Center for Turbulent Research, Stanford, 2006).

${ }^{28}$ J. Boudet, J. Caro, L. Shao, and E. Lévêque, "Numerical studies towards practical large-eddy simulation,” J. Therm. Sci. 16, 328 (2007).

${ }^{29}$ R. D. Moser, J. Kim, and N. N. Mansour, "Direct numerical simulation of turbulent channel flow up to $\operatorname{Re}_{\tau}=590$," Phys. Fluids 11, 943 (1999).

${ }^{30}$ F. Ducros, P. Comte, and M. Lesieur, "Large-eddy simulation of transition to turbulence in a boundary layer developing spatially over a flat plate," J. Fluid Mech. 326, 1 (1996).

${ }^{31}$ P. Sagaut, P. Comte, and F. Ducros, "Filtered subgrid-scale models," Phys. Fluids 12, 233 (2000).

${ }^{32}$ W. Rodi, "Large-eddy simulations of the flow past bluff bodies: State-ofthe art," JSME 41, 361 (1998).

${ }^{33} \mathrm{M}$. Breuer, "A challenging test case for large eddy simulation: High Reynolds number circular cylinder flow," Int. J. Heat Fluid Flow 21, 648 (2000).

${ }^{34}$ C. H. K. Williamson, "Vortex dynamics in the cylinder wake," Annu. Rev. Fluid Mech. 28, 477 (1996).

${ }^{35}$ S. Szepessy and P. W. Bearman, "Aspect ratio and end plate effects on vortex shedding from a circular cylinder," J. Fluid Mech. 234, 191 (1992).

${ }^{36}$ H. Nishimura and Y. Taniike, "Aerodynamic characteristics of fluctuating forces on a circular cylinder,” J. Wind. Eng. Ind. Aerodyn. 89, 713 (2001).

${ }^{37}$ M. M. Zdravkovich, Flow Around Circular Cylinders (Oxford University Press, Oxford, 2002).

${ }^{38}$ B. Cantwell and D. Coles, "An experimental study of entrainment and transport in the turbulent near wake of a circular cylinder," J. Fluid Mech. 136, 321 (1983).

${ }^{39}$ E. Achenbach, "Distribution of local pressure and skin friction around a circular cylinder in cross-flow up to Re $=5 \times 10^{6}$," J. Fluid Mech. 34, 625 (1968).

${ }^{40}$ J. H. Gerrard, "An experimental investigation of the oscillating lift and drag of a circular cylinder shedding turbulent vortices," J. Fluid Mech. 11, 244 (1961).

${ }^{41}$ C. Norberg, "Fluctuating lift on a circular cylinder: Review and new measurements," J. Fluids Struct. 17, 57 (2003).

${ }^{42}$ S. Yokuda and B. R. Ramaprian, "The dynamics of flow around a cylinder at subcritical Reynolds numbers," Phys. Fluids A 2, 784 (1990).

${ }^{43}$ E. A. Anderson and A. A. Szewczyk, "Effects of a splitter plate on the near wake of a circular cylinder in 2 and 3-dimensional flow configurations," Exp. Fluids 23, 161 (1997).

${ }^{44}$ J. P. Batham, "Pressure distributions on circular cylinders at critical Reynolds numbers," J. Fluid Mech. 57, 209 (1973).

${ }^{45}$ H. Djeridi, M. Braza, R. Perrin, G. Harran, E. Cid, and S. Cazin, "Nearwake turbulence proprieties around a circular cylinder at high Reynolds number," Flow, Turbul. Combust. 71, 19 (2003).

${ }^{46}$ T. S. Lund, "On the use of discrete filters for large eddy simulation," Proceedings of the Summer Program 1997 (Center for Turbulent Research, Stanford, 1997), pp. 83-95. 